\title{
A derivative free globally convergent method and its deformations
}

Received: 20 July 2020 / Accepted: 29 March 2021 / Published online: 1 May 2021

(C) The Author(s) 2021

\begin{abstract}
The motive of the present work is to introduce and investigate the quadratically convergent Newton's like method for solving the non-linear equations. We have studied some new properties of a Newton's like method with examples and obtained a derivative-free globally convergent Newton's like method using forward difference operator and bisection method. Finally, we have used various numerical test functions along with their fractal patterns to show the utility of the proposed method. These patterns support the numerical results and explain the compactness regarding the convergence, divergence and stability of the methods to different roots.
\end{abstract}

\section{Mathematics Subject Classification $\quad 65 \mathrm{H} 04 \cdot 65 \mathrm{H} 05$}

\section{Introduction}

In science and technology, most of the time a situation arises when the central problem ultimately reduces to the problem where we have to solve the nonlinear and transcendental equation, of the type

$$
F(z)=0 .
$$

Here $\mathrm{F}$ is a function or a mapping $F: X \rightarrow X$, where $\mathrm{X}$ equals the real or complex numbers. Due to the unavailability of the accurate methods for finding the solutions of (1), we use numerical iterative methods such as Newton's method, secant method, and Chebyshev method, etc. to obtain the approximate solution. Among all the iterative methods Newton's method is the most popular and basic method to find the roots of nonlinear Eq. (1) and is defined as follows

$$
z_{n+1}=z_{n}-\frac{F\left(z_{n}\right)}{F^{\prime}\left(z_{n}\right)}, \quad n=0,1,2, \ldots
$$

Newton's method (2) converges quadratically to simple zeros and linearly to multiple zeros. Its various modifications and refinement have been introduced in the literature to accelerate it or to rectify it with the use of added evaluations of functions, derivatives, and changes in the points of iterations [10,15,17,21]. In 2000, Weerakoon et al. [20] developed a method known as trapezoidal Newton's method or arithmetic mean Newton's method while in 2004, Ozban [3] gave some new variants of Newton's method based on harmonic mean and midpoint.

M. K. Singh · A. K. Singh $(\bowtie)$

Department of Mathematics, Institute of Science, Banaras Hindu University, Varanasi 221005, India

E-mail: aksingh9@gmail.com

M. K. Singh

E-mail: manoj07777@gmail.com 
In 2009, Singh [16] gave a six-order variant of Newton's method for solving (1) and in 2000, Wu et al. [23] developed a second-order method for solving nonlinear equations in weak condition as follows

$$
z_{n+1}=z_{n}-\frac{F\left(z_{n}\right)}{\lambda F\left(z_{n}\right)+F^{\prime}\left(z_{n}\right)}, \quad n=0,1,2, \ldots,
$$

where $\lambda \in R$ and $\lambda \in(-\infty,+\infty)$. Further, Wang [8] established a third-order variant of Newton's method for solving nonlinear equations under the weak condition in 2010. Kou [11] gave a third-order modification of Newton's method for solving the system of nonlinear equations. The study of the above methods is only modifying Newton's method by increasing its order of convergence or making it applicable in weak conditions. These methods are not explaining several questions related to the solution, which are as follows:

1. How does the starting point related to the roots?

2. Why does the iteration process oscillates to the same starting point?

3. Why does the iteration process fails, diverges or converges to an undesired root?

4. What is the importance of the denominator term in the method?

Although, several researchers have modified Newton's method in different spaces (e.g., $R, R^{n}, C$, and Banach space). But the problem with the solution and convergence persists in the study of Newton's like method until we study the numerical results along with the study of dynamics of Newton's like method. In this paper, we have explained the convergence and stability of the methods by describing the dynamics of Newton's like method. The dynamics of the discrete version of Newton's method show some beautiful fractal patterns. These patterns contain the basins of attraction around all the roots. Starting with any point in this basin is a guarantee of the convergence to the corresponding roots.

In Sect. 2, we have given some preliminary definitions. In Sect. 3, we have described the proposed method and done its convergence analysis. In Sect. 4, we have considered the continuous version of Newton's like proposed method, while in Sect. 5, we have explained the derivative free version of our proposed method using the forward difference operator with some numerical examples. In Sect. 6, we have obtained a globally convergent method using the bisection method. Finally, the numerical results of Sect. 5 have been supported by different fractal patterns.

\section{Preliminary}

Definition 2.1 (see $[9,14]$ ) Let $z_{n}, z_{n-1}, \ldots, z_{n-k+1}$ be $k$ approximations to the root of (1). Then an iterative method defined as

$$
z_{n+1}=g\left(z_{n}, z_{n-1}, \ldots, z_{n-k+1}\right),
$$

is called multi-point iteration method and the function $g$ is called multi-point iteration function.

Remark For $k=1$, we get the one point iteration method e.g.,

$$
z_{n+1}=g\left(z_{n}\right) .
$$

Definition 2.2 (see $[9,14]$ ) A sequence of iterates $\left\{z_{n} \mid n \geq 0\right\}$ is said to converge to a limit $z^{*}$ with order $p \geq 0$ if

$$
\lim _{z_{n} \rightarrow z^{*}} \frac{\left|z_{n+1}-z^{*}\right|}{\left|z_{n}-z^{*}\right|^{p}}=C
$$

where $C>0$ is known as asymptotic error constant. In case of $p=1$, the method is said to Converge linearly, and in case of $p=2$, the method is said to converge quadratically (see Eqs. (12) and (25)).

Remark Let $e_{n}=z_{n}-z^{*}$. Then an error equation of $p^{t h}$ order method can be written as $e_{n+1}=C e_{n}^{p}+$ $O\left(e_{n}^{p+1}\right)$.

Now, we will define the following definitions in the extended complex plane. 
Definition 2.3 (see [1,18]) Let I be a subset of of the complex numbers C. Let us consider $\mathrm{g}: \mathrm{I} \rightarrow \mathrm{C}$ be a rational map on the Riemann sphere. Then a point $z_{0}$ is said to be a fixed point of $\mathrm{g}$ if

$$
g\left(z_{0}\right)=z_{0} \text {. }
$$

Again for any point $z \in C$, the Orbit of the point $\mathrm{z}$ can be defined as the set

$$
\operatorname{Orb}(z)=\left\{z, g(z), g^{2}(z), \ldots, g^{n}(z), \ldots\right\} .
$$

Definition 2.4 (see $[1,18]$ ) A periodic point $z_{0}$ is said to be of period $\mathrm{k}$ if $\exists$ a smallest positive integer $\mathrm{k}$ i.e., $g^{k}\left(z_{0}\right)=z_{0}$.

Remark If $z_{0}$ is periodic point of period $\mathrm{k}$, then clearly it is a fixed point for $g^{k}$.

Definition 2.5 (see [1,18]) Let $z^{*}$ be a zero of the function $F$. Then the basin of attraction of the zero $z^{*}$ is defined as the set of collection of all initial approximations $z_{0}$ such that any numerical iterative method with $z_{0}$ converges to $z^{*}$. It can be written as

$$
B\left(z^{*}\right)=\left[z_{0}: z_{n}=g^{n}\left(z_{0}\right) \text { converges } \rightarrow z^{*}\right] .
$$

Here $g^{n}$ is any fixed point iterative method.

Remark For example in case of Newton's method

$$
\begin{aligned}
& z_{n+1}=g\left(z_{n}\right), \\
& g\left(z_{n}\right)=z_{n}-\frac{F\left(z_{n}\right)}{F^{\prime}\left(z_{n}\right)}, \quad n=0,1,2, \ldots .
\end{aligned}
$$

We can write the basin of attraction of the zero $z^{*}$ for the Newton's method as follows:

$$
B\left(z^{*}\right)=\left[z_{0}: z_{n}=g^{n}\left(z_{0}\right) \text { converges } \rightarrow z^{*}\right] .
$$

Definition 2.6 (see $[1,18]$ ) The Julia set of a nonlinear map $g(z)$ is denoted as $J(g)$ and is defined as a set consisting of the closure of its repelling periodic points. The complement of Julia set $\mathrm{J}(\mathrm{g})$ is called as the Fatou set $\mathrm{f}(\mathrm{g})$.

Remark (i) Some times Julia set of a nonlinear map may also be defined as the common boundary shared by basins of the roots and the Fatou set may also be defined as the set which contains the basin of attraction.

(ii) Fractals are a very complicated phenomenon that may be defined as a self-similar surprising geometric object which repeated at every small scale [2].

\section{Description of method}

Let $\alpha$ be a zero of a function (or a root of a non-linear equation) $F(z)=0$ and $F: I \subset \Re \rightarrow \Re$ be a two times differentiable function. Then the numerical solution can be written as

$$
F(z)=F\left(z_{n}\right)+\int_{z_{n}}^{z} F^{\prime}(t) \mathrm{d} t
$$

Approximating the integral by $\left(z-z_{n}\right) F^{\prime}\left(z_{n}\right)$ and then putting $z=\alpha$ in Eq. (6), we get

$$
0 \approx F\left(z_{n}\right)+\left(\alpha-z_{n}\right) F^{\prime}\left(z_{n}\right)
$$

therefore, an approximation $y_{n}$ to $\alpha$ can be written as

$$
y_{n}=z_{n}-\frac{F\left(z_{n}\right)}{F^{\prime}\left(z_{n}\right)}, \quad n=0,1,2, \ldots
$$

On replacing $F^{\prime}\left(z_{n}\right)$ with $\lambda_{n} F\left(z_{n}\right)+F^{\prime}\left(z_{n}\right)$, we may obtain

$$
z_{n+1}=z_{n}-\frac{F\left(z_{n}\right)}{\lambda_{n} F\left(z_{n}\right)+F^{\prime}\left(z_{n}\right)}, \quad n=0,1,2, \ldots
$$

where $\left|\lambda_{n}\right| \in[0,1]$ is an arbitrary parameter chosen in such a way that the denominator term in (7) does not vanish. Newton's like method (7) is similar to the method given in Eq. (3) by Wu et al. [23] for $n=0,1,2, \ldots$ The existence and uniqueness of the zero are given by the following propositions. 
Proposition 3.1 [23] If the function $F(z)$ satisfies the following two conditions:

(i) $F \in C^{1}[a, b]$ and

(ii) $F^{\prime}(z)+\lambda F(z) \neq 0$, for all $z \in[a, b]$,

then, there exists at most one zero in $[a, b]$ for the nonlinear function $F(z)$.

Proof We define the auxiliary function $g(z)=\exp (\lambda z) F(z)$. Then, we have

$$
g^{\prime}(z)=\exp (\lambda z)\left\{\lambda F(z)+F^{\prime}(z)\right\} .
$$

Let there exist two different zeros of the function $F(z)$ in $[a, b]$. Then by using the Rolle's theorem, we conclude that there exist at least one real number $\xi \in(a, b)$ such that $g^{\prime}(\xi)=0$, which contradicts that $\lambda F(z)+F^{\prime}(z) \neq 0$ in $[a, b]$.

Proposition 3.2 [23] If the function $F(z)$ satisfies the conditions:

(i) $F \in C^{1}[a, b]$,

(ii) $F(a) F(b)<0$ and

(iii) $F^{\prime}(z)+\lambda F(z) \neq 0$, for all $z \in[a, b]$,

then, there exists a unique zero in $[a, b]$ for the nonlinear function $F(z)$.

Proof For $g(z)=\exp (\lambda z) F(z)$, we have $g(a) g(b)<0$, then by Proposition 3.1 and intermediate value theorem, $g(z)=0$ has a unique zero under the given condition, so does the function $F(z)$ also has a unique zero in $[a, b]$.

\subsection{Convergence analysis}

Theorem 3.1 Let $I:=[a, b]$ be a compact interval in $\Re$ and $F: I \rightarrow \Re$ be a function such that

(i) $F(a) F(b)<0$,

(ii) $z^{*} \in I$ be a simple zero of a function $F$,

(iii) $F$ is twice differentiable function on I and

(iv) $\lambda F(z)+F^{\prime}(z) \neq 0, \forall z \in N\left(z^{*}\right)$, where $N\left(z^{*}\right)$ is neighborhood of $z^{*}$ and $|\lambda| \leq 1$. Then, the Newton's like method defined by (7) is quadratically convergent to the zero of $F$ locally.

Proof Let $z^{*} \in I$ be a simple zero of a function $F, e_{n}:=z_{n}-z^{*}$ and $A_{k}:=\left(\frac{1}{k !}\right) \frac{F^{(k)}\left(z^{*}\right)}{F^{\prime}\left(z^{*}\right)}$. Applying Taylor's expansion of $F\left(z_{n}\right)$ aound $z^{*}$ and using $F\left(z^{*}\right)=0$, we get

$$
\begin{aligned}
& F\left(z_{n}\right)=F^{\prime}\left(z^{*}\right)\left[e_{n}+A_{2} e_{n}^{2}+A_{3} e_{n}^{3}+O\left(e_{n}^{4}\right)\right], \\
& F^{\prime}\left(z_{n}\right)=F^{\prime}\left(z^{*}\right)\left[1+2 A_{2} e_{n}+3 A_{3} e_{n}^{2}+4 A_{4} e_{n}^{3}+O\left(e_{n}^{4}\right)\right] .
\end{aligned}
$$

Now from Eqs. (8) and (9), we get

$$
\begin{aligned}
\lambda_{n} F\left(z_{n}\right)+F^{\prime}\left(z_{n}\right)= & F^{\prime}\left(z^{*}\right)\left[1+\left(2 A_{2}+\lambda_{n}\right) e_{n}+\left(3 A_{3}+\lambda_{n} A_{2}\right) e_{n}^{2}\right. \\
& \left.+\left(4 A_{4}+\lambda_{n} A_{2}\right) e_{n}^{3}+O\left(e_{n}^{4}\right)\right],
\end{aligned}
$$

and from Eqs. (8) and (10), we get

$$
\frac{F\left(z_{n}\right)}{\lambda_{n} F\left(z_{n}\right)+F^{\prime}\left(z_{n}\right)}=e_{n}-\left(A_{2}+\lambda_{n}\right) e_{n}^{2}+\left(2 A_{2}^{2}+2 A_{2} \lambda_{n}+\lambda_{n}^{2}-2 A_{3}\right) e_{n}^{3}+O\left(e_{n}^{4}\right) .
$$

Hence from proposed method (7), we obtain,

$$
z_{n+1}=z^{*}+\left(A_{2}+\lambda_{n}\right) e_{n}^{2}-\left(2 A_{2}^{2}+2 A_{2} \lambda_{n}+\lambda_{n}^{2}-2 A_{3}\right) e_{n}^{3}+O\left(e_{n}^{4}\right) .
$$

Now, using the fact that $z_{n+1}=z^{*}+e_{n+1}$, we get from (11)

$$
e_{n+1}=\left(A_{2}+\lambda_{n}\right) e_{n}^{2}-\left(2 A_{2}^{2}+2 A_{2} \lambda_{n}+\lambda_{n}^{2}-2 A_{3}\right) e_{n}^{3}+O\left(e_{n}^{4}\right) .
$$

Hence, proposed method converges quadratically (see (4)). 


\section{Continuous version of the Newton's like method}

Let us consider the damped version of Newton's like method (7) which will works in case of Newton's method fails.

$$
z_{n+1}:=z_{n}-\frac{F\left(z_{n}\right)}{\lambda_{n} F\left(z_{n}\right)+F^{\prime}\left(z_{n}\right)}, \quad n=0,1,2, \ldots
$$

Here $\lambda_{n} \in[-1,1]$ is an arbitrary parameter chosen in such a way that the denominator in (13) does not vanish. Hence above modified Newton method will work when Newton's method get fails. Therefore, considering the damped version of the above modified Newton method (13), we have

$$
z_{n+1}-z_{n}=-h \frac{F\left(z_{n}\right)}{\lambda_{n} F\left(z_{n}\right)+F^{\prime}\left(z_{n}\right)}, \quad n=0,1,2, \ldots
$$

Hence the damped version of the above modified Newton method (13) will work even when damped version of Newton method get fails i.e., damped modified Newton method (14) will have a larger basin of attraction and less chaotic behavior than the damped Newton's method for $h>0$. Consequently, the continuous version of the above modified Newton's method (13) will transport any initial point to the solution with a faster speed of convergence and a larger basins of attraction.

$$
\begin{aligned}
& z(t+h)-z(t)=-h \frac{F(z(t))}{\lambda_{n} F(z(t))+F^{\prime}(z(t))}, \\
& \lim _{h \rightarrow 0} \frac{z(t+h)-z(t)}{h}=-\frac{F(z(t))}{\lambda_{n} F(z(t))+F^{\prime}(z(t))} .
\end{aligned}
$$

From above, we get

$$
\left\{\begin{array}{l}
\frac{d z}{d t}=-\frac{F(z(t))}{\lambda_{n} F(z(t))+F^{\prime}(z(t))} \\
z(0)=z_{0}
\end{array}\right.
$$

Equation (15) is the continuous version of modified Newton's like method (13). Solving the above initial value problem (15) using Euler's like method will give

$$
z_{n+1}:=z_{n}-\frac{h F\left(z_{n}\right)}{\lambda_{n} F\left(z_{n}\right)+F^{\prime}\left(z_{n}\right)}, \quad n=0,1,2, \ldots
$$

Here $z_{n}$ is an numerical approximation to the solution $z(t)$ at $t=n h$, thus when the step size $h=1$, an improved version of Euler's like method produces an improved Newton's like method (13).

\section{Quadratic convergence iterative formula without derivative (discrete case)}

Let us consider the iterative formula (7) with forward, backward, and central difference operators respectively. Then we have the formula (7)

$$
y_{n}=z_{n}-\frac{F\left(z_{n}\right)}{\lambda_{n} F\left(z_{n}\right)+F^{\prime}\left(z_{n}\right)}, \quad n=0,1,2, \ldots,
$$

where

$$
F^{\prime}\left(z_{n}\right)=\lim _{z \rightarrow z_{n}} \frac{F(z)-F\left(z_{n}\right)}{z-z_{n}}
$$

Now, approximating $F^{\prime}\left(z_{n}\right)$ by using the forward difference operator with the fact that $\lim _{z_{n} \rightarrow z^{*}} F\left(z_{n}\right)=0$, we have 


$$
\begin{aligned}
F^{\prime}\left(z_{n}\right) & =\lim _{z_{n} \rightarrow z^{*}} \frac{F\left(z_{n}+F\left(z_{n}\right)\right)-F\left(z_{n}\right)}{F\left(z_{n}\right)}, \\
F^{\prime}\left(z_{n}\right) & \approx \frac{F\left(z_{n}+F\left(z_{n}\right)\right)-F\left(z_{n}\right)}{F\left(z_{n}\right)} .
\end{aligned}
$$

We put this value of $F^{\prime}\left(z_{n}\right)$ in Eq. (16) to get

$$
y_{n} \approx z_{n}-\frac{F\left(z_{n}\right)^{2}}{\lambda_{n} F\left(z_{n}\right)^{2}+F\left(z_{n}+F\left(z_{n}\right)\right)-F\left(z_{n}\right)}, \quad n=0,1,2, \ldots
$$

Similarly using backward, and central difference operators in (16) we get (18) and (19) respectively.

$$
\begin{aligned}
& y_{n} \approx z_{n}-\frac{F\left(z_{n}\right)^{2}}{\lambda_{n} F\left(z_{n}\right)^{2}+F\left(z_{n}\right)-F\left(z_{n}-F\left(z_{n}\right)\right)}, \quad n=0,1,2, \ldots \\
& y_{n} \approx z_{n}-\frac{F\left(z_{n}\right)^{2}}{\lambda_{n} F\left(z_{n}\right)^{2}+F\left(z_{n}+F\left(z_{n}\right)\right)-F\left(z_{n}-F\left(z_{n}\right)\right)}, \quad n=0,1,2, \ldots
\end{aligned}
$$

Now we will consider the proposed iteration formula with forward difference operator i.e., (17) for calculating the simple root of a nonlinear algebraic Eq. (1), the selection of the parameter $\lambda_{n}$ in proposed method (17) plays a very crucial part in the convergence. Here we select the parameter $\lambda_{n}\left(\left|\lambda_{n}\right| \in[0,1]\right)$ in such a way that the denominator of the iterative method (17) is against vanishing. In particular, let $\lambda_{n}=\operatorname{sign}\left(F\left(z_{n}+F\left(z_{n}\right)\right)-F\left(z_{n}\right)\right)$. Hence, the iteration method (17) will be as follows:

$$
\begin{aligned}
& z_{n+1} \approx z_{n}-\frac{F\left(z_{n}\right)^{2}}{\lambda_{n} F\left(z_{n}\right)^{2}+F\left(z_{n}+F\left(z_{n}\right)\right)-F\left(z_{n}\right)}, \quad n=0,1,2, \ldots . \\
& \lambda_{n}=\left\{\begin{aligned}
1, & \text { if sign }\left(F\left(z_{n}+F\left(z_{n}\right)\right)-F\left(z_{n}\right)\right) \geq 0, \\
-1, & \text { if sign }\left(F\left(z_{n}+F\left(z_{n}\right)\right)-F\left(z_{n}\right)\right)<0 .
\end{aligned}\right.
\end{aligned}
$$

Let Eq. (1) has roots (zeros of $F$ ) with multiplicity greater than one. Then we have

$$
M(z)=\frac{F(z)}{F^{\prime}(z)}=\frac{F\left(z_{n}\right)^{2}}{F\left(z_{n}+F\left(z_{n}\right)\right)-F\left(z_{n}\right)} .
$$

Now, the roots with multiplicity greater than one of non linear algebraic Eq. (1) i.e., $F(z)=0$ are transformed into the simple roots of $M(z)=0$. Hence, the iteration formula (20) will be defined as follows:

$$
z_{n+1} \approx z_{n}-\frac{M\left(z_{n}\right)^{2}}{\lambda_{n} M\left(z_{n}\right)^{2}+M\left(z_{n}+M\left(z_{n}\right)\right)-M\left(z_{n}\right)}, \quad n=0,1,2, \ldots
$$

It is at least quadratically convergent for the roots with multiplicity greater than one, where $\lambda_{n}$ is a parameter belongs to $\Re$ such that $|\lambda| \leq 1$.

The analysis of convergence of the proposed method (20) is given in the following theorem.

Theorem 5.1 Let $I:=[a, b]$ be a compact interval in $\Re$ and $F: I \rightarrow \Re$ be a function such that

(i) $F(a) F(b)<0$,

(ii) $z^{*} \in I$ be a simple zero of a function $F$,

(iii) $F$ is twice differentiable function on $I$ and $F^{\prime} \neq 0$,

(iv) $\lambda F(z)+F^{\prime}(z) \neq 0, \forall z \in N\left(z^{*}\right)$, where $N\left(z^{*}\right)$ is a neighborhood of $z^{*}$ and $|\lambda| \leq 1$. Then, the iterative derivative free method defined by (20) is quadratically convergent to the zero of $F(z)$ locally.

Proof Let $z^{*} \in I$ be a simple zero of a function $F, e_{n}:=z_{n}-z^{*}$ and $A_{k}:=\left(\frac{1}{k !}\right) \frac{F^{(k)}\left(z^{*}\right)}{F^{\prime}\left(z^{*}\right)}$. Applying Taylor's expansion of $F\left(z_{n}\right)$ around $z^{*}$ and using $F\left(z^{*}\right)=0$, we get

$$
\begin{aligned}
& F\left(z_{n}\right)=F^{\prime}\left(z^{*}\right)\left[e_{n}+A_{2} e_{n}^{2}+A_{3} e_{n}^{3}+O\left(e_{n}^{4}\right)\right], \\
& F\left(z_{n}+F\left(z_{n}\right)\right)=A_{1}\left(1+A_{1}\right) e_{n}+\left(A_{1}+\left(1+A_{1}\right)^{2}\right) A_{2} e_{n}^{2} \\
& +\left(2\left(1+A_{1}\right) A_{2}^{2}+A_{1} A_{3}+\left(1+A_{1}\right)^{3}+A_{3}\right) e_{n}^{3}+O\left(e_{n}^{4}\right) .
\end{aligned}
$$


Now, using Eqs. (22) and (23) in (20), we get

$$
z_{n+1}=z^{*}+\left(A_{2}+\lambda_{n}\right) e_{n}^{2}-\left(2 A_{2}^{2}+2 A_{2} \lambda_{n}+\lambda_{n}^{2}-2 A_{3}\right) e_{n}^{3}+O\left(e_{n}^{4}\right) .
$$

Hence, using the fact that $z_{n+1}=z^{*}+e_{n+1}$, we get from (24)

$$
e_{n+1}=\left(A_{2}+\lambda_{n}\right) e_{n}^{2}-\left(2 A_{2}^{2}+2 A_{2} \lambda_{n}+\lambda_{n}^{2}-2 A_{3}\right) e_{n}^{3}+O\left(e_{n}^{4}\right) .
$$

Therefore, the proposed derivative-free method (20) converges quadratically. From Eqs. (12) and (25), it is clear that the convergence equations are the same for both the derivative method (7) and the derivative-free method (20).

\subsection{Numerical results (discrete case)}

In this section, we have considered some test examples to show the efficiency of the proposed new derivativefree method (20). Here, we have considered the proposed new method with forward difference operator (20) at $\lambda=0.5$ and central difference operator, backward difference operator (Wu et. al. [23]), Newton's method and Steffensen's method [23].

\section{Example 5.1}

$$
F_{1}=\log (z)
$$

It has a simple zero 1.00000. Stopping criteria has been taken as $\left|F\left(z_{n}\right)\right|<7.10 e(-17)$. It is clear from Table 1, that the starting with the point $z_{0}=5$, the proposed method with forward difference operator takes a minimum number of iterations and least cpu time in computation of the zero, while Newton's method and Steffensen's method diverges.

\section{Example 5.2}

$$
F_{2}=\arctan (z)
$$

It has two simple zeros one for the forward operator is $3.040261026364800 e-010$ and other for the central operator is $2.522900892965922 e-018$. Stopping criteria has been taken as $\left|F\left(z_{n}\right)\right|<(1.77) e(-18)$. It is clear from the Table 2 that starting with the initial approximation $z_{0}=5$, the proposed method with forward difference operator takes a minimum number of iterations and less cpu time in computation of the zero, while Newton's method and Steffensen's method diverges.

\section{Example 5.3}

$$
F_{3}=z+1-\exp (\sin (z))
$$

It has a simple zero 1.69681238680975 . The stopping criteria has been taken as $\left|F\left(z_{n}\right)\right|<3.02 e(-16)$. From Table 3, it is clear that the starting with $z_{0}=4$, the proposed method takes a minimum number of iterations and least cpu time in computation of the zero, while Newton's method and Steffensen's method diverges.

Table 1 Comparison of proposed method with different methods

\begin{tabular}{llllll}
\hline$z_{0}=5$ & New forward & Central & Backward & Steffensen's & Newton \\
\hline Iterations & 07 & 55 & 10 & div & div \\
Cpu Time & 0.01560 & 0.01560 & 0.07800 & - & - \\
\hline
\end{tabular}

Table 2 Comparison of proposed method with different methods

\begin{tabular}{llllll}
\hline$z_{0}=5$ & New forward & Central & Backward & Steffensen's & Newton \\
\hline Iterations & 09 & 64 & 11 & div & div \\
Cpu Time & 0.04680 & 0.70200 & 0.04680 & - & - \\
\hline
\end{tabular}


Table 3 Comparison of proposed method with different methods

\begin{tabular}{llllll}
\hline$z_{0}=4$ & New forward & Central & Backward & Steffensen's & Newton \\
\hline Iterations & 07 & 54 & 07 & div & div \\
Cpu Time & 0.07801 & 0.14040 & 0.14040 & - & - \\
\hline
\end{tabular}

\section{Convergence in diameter for the method (discrete case)}

We have discussed the quadratically convergent derivative-free iterative method (20) and (21) for finding simple and multiple roots of nonlinear algebraic Eq. (1) respectively. But both the method does not have nice asymptotic convergence properties of the diameters $\left\{b_{n}-a_{n}\right\}$. Alefeld and Potra [4,5] have developed a method with nice asymptotic convergence of the diameter using bisection method. Further, Wu and Fu [23] also studied a method with asymptotic convergence of the diameter. It motivates us to consider an iterative formula with a parameter $\lambda$ and stepsize $h$ of the form as follows

$$
y_{n} \approx z_{n}-\frac{h_{n} F\left(z_{n}\right)^{2}}{\lambda_{n} F\left(z_{n}\right)^{2}+F\left(z_{n}+h_{n} F\left(z_{n}\right)\right)-F\left(z_{n}\right)}, \quad n=0,1,2, \ldots,
$$

where $z_{0} \in[a, b]$ and $\lambda_{n} \in R,\left|\lambda_{n}\right| \leq 1$ and $h_{n}>0$.

Theorem 6.1 Let $I:=[a, b]$ be a compact interval in $\Re$ and $F: I \rightarrow \Re$ be a function such that

(i) $F(a) F(b)<0$,

(ii) $z^{*} \in I$ be a simple zero of a function $F$,

(iii) $F$ is twice differentiable function on $I$ and $F^{\prime} \neq 0$

(iv) $\lambda F(z)+F^{\prime}(z) \neq 0, \forall z \in N\left(z^{*}\right)$, where $N\left(z^{*}\right)$ is neighborhood of $z^{*}$ and $|\lambda| \leq 1$. Then, the Newton's like iterative method defined by (29) is at least quadratically convergent to the zero $z^{*}$ of $F(z)$ locally for $h_{n}>0$.

Proof The proof of Theorem 6.1 is similar to that of Theorem 5.1 and will be omitted.

To achieve the nice asymptotic convergence of the diameters $\left\{b_{n}-a_{n}\right\}$, let $h_{n}=\left(b_{n}-a_{n}\right) /\left(2\left|F\left(z_{n}\right)\right|\right)$, see $([4,5])$, we have the following iterative formula:

$$
z_{n+1} \approx z_{n}-\frac{\left(b_{n}-a_{n}\right)\left|F\left(z_{n}\right)\right|}{2\left(\lambda_{n} F\left(z_{n}\right)^{2}+F\left(\left(a_{n}+b_{n}\right) / 2\right)-F\left(z_{n}\right)\right)}, \quad n=0,1,2, \ldots
$$

Here $z_{0}=a$ or $b$ and $\lambda_{n}=\operatorname{sign}\left(F\left(\left(a_{n}+b_{n}\right) / 2\right)-F\left(z_{n}\right)\right)$.

For the special case of $\lambda_{n}=0$, we have the iterative formula as follows

$$
z_{n+1} \approx z_{n}-\frac{\left(b_{n}-a_{n}\right)\left|F\left(z_{n}\right)\right|}{2\left(F\left(\left(a_{n}+b_{n}\right) / 2\right)-F\left(z_{n}\right)\right)}, \quad n=0,1,2, \ldots
$$

Now, we will combine the formula (30) with the bisection method for constructing the following new algorithm defined as follows:

\section{New algorithm for convergence in diameter (ACD)}

Suppose $n=0,1,2, \ldots$ Then, we have

1. Let $d_{n}=\frac{a_{n}+b_{n}}{2}$.

2. Compute $F\left(d_{n}\right)$, if $F\left(d_{n}\right)=0$, then print $d_{n}$ and stop.

3. If $F\left(d_{n}\right) \neq 0$ and $\operatorname{sign}\left(F\left(d_{n}\right)\right)=\operatorname{sign}\left(F\left(a_{n}\right)\right)$, then take $a_{n}^{\prime}=d_{n}, b_{n}^{\prime}=b_{n}$, otherwise $a_{n}^{\prime}=a_{n}, b_{n}^{\prime}=d_{n}$.

4. Let $w_{n}=z_{n}-\frac{\left(b_{n}-a_{n}\right)\left|F\left(z_{n}\right)\right|}{2\left(\lambda_{n} F\left(z_{n}\right)^{2}-F\left(z_{n}\right)+F\left(d_{n}\right)\right)}$. Since $n=0,1,2, \ldots$, hence $z_{n}$ will be initialized as $z_{0}=a_{0}$ or $b_{0}$.

5. If $w_{n} \in\left[a_{n}^{\prime}, b_{n}^{\prime}\right]$, then if $F\left(a_{n}^{\prime}\right) f\left(w_{n}\right)<0$, then $\left[a_{n+1}, b_{n+1}\right]=\left[a_{n}^{\prime}, w_{n}\right]$, else $\left[a_{n+1}, b_{n+1}\right]=$ $\left[w_{n}, b_{n}^{\prime}\right], z_{n+1}=w_{n}$.

6. If $w_{n} \notin\left[a_{n}^{\prime}, b_{n}^{\prime}\right]$, then $\left[a_{n+1} b_{n+1}\right]=\left[a_{n}^{\prime}, b_{n}^{\prime}\right]$.

7. If $\left|F\left(z_{n}\right)\right|<\varepsilon_{1}$ or $b_{n+1}-a_{n+1}<\varepsilon_{2}$, then print $z_{n+1}$ and stop. 
Here $\varepsilon_{1}$ and $\varepsilon_{2}$ are taken precision during the solution. Repeating algorithm (ACD) by taking $n+1$ in place of $n$, we will generate a sequence $z_{n}$ of iterations and sequence of diameters $\left(b_{n}-a_{n}\right)$.

Theorem 6.2 Let $F:[a, b] \rightarrow \Re$ be a function such that

1. F is continuous,

2. $F(a) F(b)<0$.

Then either a zero of function $F(z)$ is obtained in a finite number of steps, or the sequence of diameter $\left(b_{n}-a_{n}\right)$ generated by new Algorithm (ACD) converges to zero.

Because of the fact that we are using the bisection method, the proof of the theorem is straightforward and will be omitted.

Theorem 6.3 Let $I:=[a, b]$ be a compact interval in $\Re$ and $F: I \rightarrow \Re$ be a function such that

1. $F \in C^{2}[a, b]$,

2. $F(a) F(b)<0$,

3. it has simple zero $z^{*} \in I$,

4. $\lambda F(z)+F^{\prime}(z) \neq 0, \forall z \in N\left(z^{*}\right)$ where $N\left(z^{*}\right)$ is neighbourhood of $z^{*}$,

5. New algorithm $(A C D)$ does not terminate after finite number of steps.

Then, the sequence of diameter $\left(b_{n}-a_{n}\right)_{n=1}^{n=\infty}$ produced by algorithm (ACD) converges $Q$ quadratically to zero, i.e., there is a constant $C$ such that

$$
b_{n+1}-a_{n+1} \leq C\left(b_{n}-a_{n}\right)^{2}, \quad n=0,1,2, \ldots
$$

and $\left(b_{n}-a_{n}\right) \rightarrow 0$, for $n \rightarrow \infty$.

Proof From Theorem 6.1, it follows that

$\lim _{n \rightarrow \infty} \frac{e_{n+1}}{e_{n}^{2}}=C^{\prime}$ or $\lim _{n \rightarrow \infty} \frac{e_{n}}{e_{n-1}^{2}}=C^{\prime}$. Now, from the definition of the limit we have

$$
\begin{aligned}
\lim _{n \rightarrow \infty} \frac{e_{n+1}-e_{n}}{\left(e_{n}-e_{n-1}\right)^{2}} & =\lim _{n \rightarrow \infty} \frac{\frac{e_{n+1}}{e_{n}}-1}{\left(e_{n}-2 e_{n-1}+\frac{e_{n-1}^{2}}{e_{n}}\right)} \\
& =\lim _{n \rightarrow \infty} \frac{\frac{e_{n}}{e_{n-1}^{2}}\left(\frac{e_{n+1}}{e_{n}^{2}} e_{n}-1\right)}{\frac{e_{n}}{e_{n-1}^{2}}\left(e_{n}-2 e_{n-1}\right)+1} \\
& =\lim _{n \rightarrow \infty} \frac{C^{\prime}(0-1)}{(0+1)}, \quad\left(n \rightarrow \infty \rightarrow e_{n} \rightarrow 0\right) \\
& =-C^{\prime} .
\end{aligned}
$$

Then, clearly there exist an integer $N^{0}$ such that $\left|\frac{e_{n+1}-e_{n}}{\left(e_{n}-e_{n-1}\right)^{2}}\right|<\left|C^{\prime}\right|+1$, and then for $n>N^{0}$ we have

$$
\left|\frac{z_{n+1}-z_{n}}{\left(z_{n}-z_{n-1}\right)^{2}}\right|<\left|C^{\prime}\right|+1 \text {. }
$$

Again from the convergence of bisection method and algorithm ACD we have

$$
d_{n}=\frac{a_{n}+b_{n}}{2}
$$

Hence, $d_{n}$ is better approximation to the root in comparison to the $z_{n}$, where $z_{0}=a$ or $b$. Therefore,

$$
\left|F\left(d_{n}\right)\right|<\left|F\left(z_{n}\right)\right|
$$

as $z_{n}$ tends to root of $F(z)=0$. From above inequality (32), there exists $0<d<1$ such that $\forall \mathrm{n} \geq N^{1}$, we have

$$
\left|F\left(d_{n}\right)\right| \leq d\left|F\left(z_{n}\right)\right|, \quad 0<d<1 .
$$


From Eq. (32), if $F\left(z_{n}\right)$ is negative then $F\left(z_{n}\right)-F\left(d_{n}\right)$ is aways negative (whether $F\left(d_{n}\right)$ is - ve or + ve) and, therefore, the sign of $F\left(z_{n}\right)-F\left(d_{n}\right)$ is same as $F\left(z_{n}\right)$. Similarly it is true for $F\left(z_{n}\right)$ is positive. Hence, it is clear that $F\left(z_{n}\right)-F\left(d_{n}\right)$ has the same sign as $F\left(z_{n}\right)$, therefore, for $n>N^{1}$, we have

$$
\frac{F\left(z_{n}\right)-F\left(d_{n}\right)}{F\left(z_{n}\right)}>0
$$

or

$$
\frac{F\left(d_{n}\right)-F\left(z_{n}\right)}{F\left(z_{n}\right)}<0
$$

Now from (30)

$$
\left(b_{n}-a_{n}\right)=\frac{2\left(z_{n+1}-z_{n}\right)\left(\lambda_{n} F\left(z_{n}\right)^{2}-F\left(z_{n}\right)+f\left(d_{n}\right)\right)}{\left|F\left(z_{n}\right)\right|},
$$

and then it follows

$$
\frac{b_{n+1}-a_{n+1}}{\left(b_{n}-a_{n}\right)^{2}}=-\frac{F\left(z_{n}\right)^{2}\left(z_{n+2}-z_{n+1}\right)\left(\lambda_{n+1} F\left(z_{n+1}\right)^{2}-F\left(z_{n+1}\right)+F\left(d_{n+1}\right)\right)}{2\left|F\left(z_{n+1}\right)\right|\left(z_{n+1}-z_{n}\right)^{2}\left(\lambda_{n} F\left(z_{n}\right)^{2}-F\left(z_{n}\right)+F\left(d_{n}\right)\right)^{2}},
$$

or

$$
\frac{b_{n+1}-a_{n+1}}{\left(b_{n}-a_{n}\right)^{2}}=\frac{F\left(z_{n}\right)^{2}\left(z_{n+2}-z_{n+1}\right)\left(F\left(z_{n+1}\right)-F\left(d_{n+1}\right)-\lambda_{n+1} F\left(z_{n+1}\right)^{2}\right)}{2\left|F\left(z_{n+1}\right)\right|\left(z_{n+1}-z_{n}\right)^{2}\left(F\left(z_{n}\right)-F\left(d_{n}\right)-\lambda_{n} F\left(z_{n}\right)^{2}\right)^{2}} .
$$

From (33), we have $\frac{\left|F\left(d_{n}\right)\right|}{\left|F\left(z_{n}\right)\right|} \leq d$ or $-\frac{\left|F\left(d_{n}\right)\right|}{\left|F\left(z_{n}\right)\right|} \geq-d$. Since $\lambda_{n}=\operatorname{sign}\left(F\left(d_{n}\right)-F\left(z_{n}\right)\right)$, hence using the fact that $\lambda_{n}$ and $F\left(z_{n}\right)$ are of opposite sign, we have

$$
\begin{aligned}
\left|\lambda_{n} F\left(z_{n}\right)\right|+1-d & \leq\left|\lambda_{n} F\left(z_{n}\right)\right|+1-\left|\frac{F\left(d_{n}\right)}{F\left(z_{n}\right)}\right| \\
& =\frac{\left|\lambda_{n} F\left(z_{n}\right)^{2}\right|+\left|F\left(z_{n}\right)\right|-\left|F\left(d_{n}\right)\right|}{\left|F\left(z_{n}\right)\right|} .
\end{aligned}
$$

Since $\lambda_{n}$ and $F\left(z_{n}\right)$ are of opposite sign, therefore, $\left|\lambda_{n} F\left(z_{n}\right)^{2}\right|+\left|F\left(z_{n}\right)\right|=\left|\lambda_{n} F\left(z_{n}\right)^{2}-F\left(z_{n}\right)\right|$ and hence from Eq. (35) we have

$$
\begin{aligned}
\left|\lambda_{n} F\left(z_{n}\right)\right|+1-d & \leq \frac{\left|\lambda_{n} F\left(z_{n}\right)^{2}-F\left(z_{n}\right)\right|-\left|F\left(d_{n}\right)\right|}{\left|F\left(z_{n}\right)\right|} \\
& \leq\left|\frac{\lambda_{n} F\left(z_{n}\right)^{2}-F\left(z_{n}\right)+F\left(d_{n}\right)}{F\left(z_{n}\right)}\right| \\
& =\left|\lambda_{n} F\left(z_{n}\right)-\left[1-\frac{F\left(d_{n}\right)}{F\left(z_{n}\right)}\right]\right| \\
& \leq\left|\lambda_{n} F\left(z_{n}\right)\right|+\left|1-\frac{F\left(d_{n}\right)}{F\left(z_{n}\right)}\right| \\
& \leq\left|\lambda_{n} F\left(z_{n}\right)\right|+1+d .
\end{aligned}
$$

From $\lim _{n \rightarrow \infty} F\left(z_{n}\right)=0$, we can select an $\epsilon=\frac{1}{2}(1-d), 0<d<1$ such that

$$
\left|F\left(z_{n}\right)-0\right|<\frac{1}{2}(1-d)
$$

or

$$
\left|F\left(z_{n}\right)\right|<\frac{1}{2}(1-d),
$$

whenever $n>N^{2}$. 
Now from inequality (36), we get

$$
\frac{\left|\lambda_{n} F\left(z_{n}\right)^{2}-F\left(z_{n}\right)+F\left(d_{n}\right)\right|}{\left|F\left(z_{n}\right)\right|}>1-d,
$$

and from inequalities (37) and (38), we get

$$
\frac{\left|\lambda_{n} F\left(z_{n}\right)^{2}-F\left(z_{n}\right)+F\left(d_{n}\right)\right|}{\left|F\left(z_{n}\right)\right|}<\frac{(1-d)}{2}+(1+d),
$$

therefore,

$$
1-d<\frac{\left|\lambda_{n} F\left(z_{n}\right)^{2}-F\left(z_{n}\right)+F\left(d_{n}\right)\right|}{\left|F\left(z_{n}\right)\right|}<\frac{(d+3)}{2} .
$$

As $\lambda_{n}$ and $F\left(z_{n}\right)$ are of opposite sign, we have

$$
\begin{aligned}
& \lambda_{n} F\left(z_{n}\right)+\frac{F\left(d_{n}\right)-F\left(z_{n}\right)}{F\left(z_{n}\right)}<0, \\
& \frac{F\left(z_{n}\right)-F\left(d_{n}\right)-\lambda_{n} F\left(z_{n}\right)^{2}}{F\left(z_{n}\right)}>0 .
\end{aligned}
$$

So we can write Eq. (39) as

$$
1-d<\frac{F\left(z_{n}\right)-F\left(d_{n}\right)-\lambda_{n} F\left(z_{n}\right)^{2}}{F\left(z_{n}\right)}<\frac{(d+3)}{2},
$$

which gives

$$
\frac{F\left(z_{n}\right)^{2}}{\left(F\left(z_{n}\right)-F\left(d_{n}\right)-\lambda_{n} F\left(z_{n}\right)^{2}\right)^{2}}<\frac{1}{(1-d)^{2}} .
$$

Now form above two Eqs. (40) and (41) we have

$$
\frac{\left|F\left(z_{n}\right)^{2}\right|\left|F\left(z_{n+1}\right)-F\left(d_{n+1}\right)-\lambda_{n+1} F\left(z_{n+1}\right)^{2}\right|}{\left|F\left(z_{n+1}\right)\right|\left|F\left(z_{n}\right)-F\left(d_{n}\right)-\lambda_{n} F\left(z_{n}\right)^{2}\right|^{2}}<\frac{(d+3)}{2(1-d)^{2}} .
$$

Therefore, using Eqs. (42) and (31) in Eq. (34), we get

$$
\frac{b_{n+1}-a_{n+1}}{\left(b_{n}-a_{n}\right)^{2}} \leq \frac{(d+3)\left(\left|C^{\prime}\right|+1\right)}{4(1-d)^{2}},
$$

where $n>N=\max \left\{N^{0}, N^{1}, N^{2}\right\}$.

For $n=0,1,2, \ldots, N$, using the definition of Bisection method, we have

$$
b_{N+1}-a_{N+1}=\frac{b_{N}-a_{N}}{2} \text {. }
$$

and hence

$$
\frac{b_{N+1}-a_{N+1}}{\left(b_{N}-a_{N}\right)^{2}}=\frac{1}{2\left(b_{N}-a_{N}\right)} .
$$

From Eqs. (43) and (44), let us soppose that

$$
C \geq \max \left\{\frac{(3+d)\left(\left|C^{\prime}\right|+1\right)}{4(1-d)^{2}}, \frac{1}{2\left(b_{N}-a_{N}\right)}\right\},
$$

Therefore, we have

$$
\frac{b_{n+1}-a_{n+1}}{\left(b_{n}-a_{n}\right)^{2}} \leq C,
$$

i.e.,

$$
b_{n+1}-a_{n+1} \leq C\left(b_{n}-a_{n}\right)^{2} \quad n=0,1,2,3, \ldots
$$

Hence, the proof is completed. 
Table 4 Comparison of proposed method with method of $\mathrm{Wu}$

\begin{tabular}{llll}
\hline Starting Interval & $z^{*}$ & New Proposed forward & Wu et al. \\
\hline$[0.5,5]$ & Iterations & 07 & 09 \\
{$[0.5,5]$} & Cpu Time & 0.07801 & 0.07801 \\
\hline
\end{tabular}

Table 5 Comparison of proposed method with method of Wu

\begin{tabular}{llll}
\hline Starting Interval & $z^{*}$ & New Proposed forward & Wu et al. \\
\hline$[-1,5]$ & Iterations & 04 & 05 \\
{$[-1,5]$} & Cpu Time & 0.17801 & 0.17801 \\
\hline
\end{tabular}

Table 6 Comparison of proposed method with method of $\mathrm{Wu}$

\begin{tabular}{llll}
\hline Starting Interval & $z^{*}$ & New Proposed forward & Wu et al. \\
\hline$[1,4]$ & Iterations & 09 & 09 \\
{$[1,4]$} & Cpu Time & 0.15501 & 0.14550 \\
\hline
\end{tabular}

\subsection{Numerical Results for Convergence in Diamters (Discrete Case)}

In this section, we present the results of some numerical tests to compare the efficiency of the proposed new algorithm (ACD) with the method of $\mathrm{Wu}$ and $\mathrm{Fu}[23]$. Here we have taken $\varepsilon_{1}:=\epsilon_{2}:=1.0 \times 10^{-15}$.

\section{Example 6.1}

$$
f_{1}=\log (z)
$$

It has a simple zero 1.00000. Starting interval has been taken as $[0.5,5]$. From the Table 4, it is clear that the proposed method with forward difference operator takes minimum number of iterations and less cpu time in computation of the root.

\section{Example 6.2}

$$
f_{2}=\arctan (z)
$$

The starting interval has been taken as $[-1,5]$. From Table 5, it is clear that the proposed method with the forward difference operator takes fewer iterations while cpu time in computation of the root is the same for both methods.

\section{Example 6.3}

$$
f_{3}=z+1-\exp (\sin (z))
$$

It has a simple zero 1.69681238680975. Starting interval has been taken as [1, 4]. It is clear from Table 6, that the proposed method takes the same number of iterations and almost equal cpu time in computing the root.

\section{Dynamical analysis of methods}

In this section, we have considered the test examples of section 5.1 (Eqs. 26-28) to discuss the basin of attraction (see Eq. (5)) of different methods of Sect. 5. The problem of finding basins of attractions for complex polynomials is also known as Cayley's problem. Basins of attraction of the zeros of function F(z) sharing a common boundary called as Julia set of $\mathrm{F}(\mathrm{z})$ [6]. Clearly, any point on the boundary of one set is also on the boundary of the other sets. Basins of attraction with this type of property are known as the Wada property, given by Japanese mathematician Konizô Yoneyama in 1917 [12]. Due to the Wada property, any neighborhood of a point on the boundary of the basin of attraction will contain different other points due to 


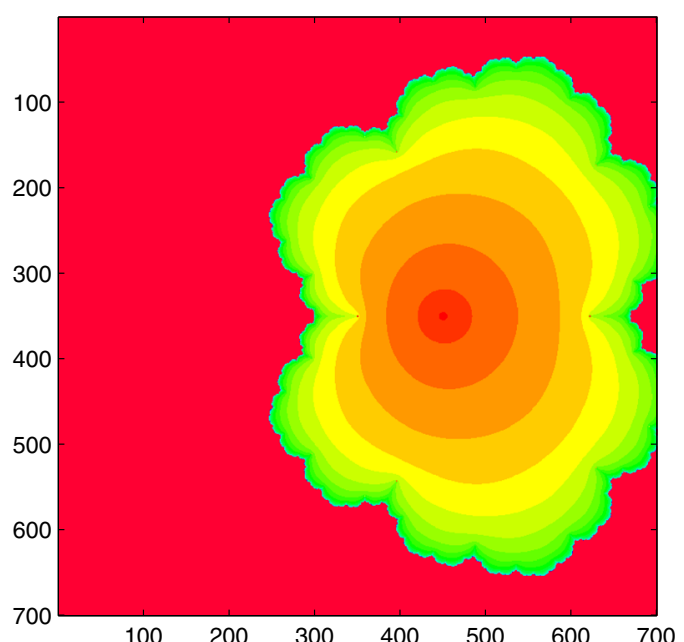

(a) Newton's method (2)

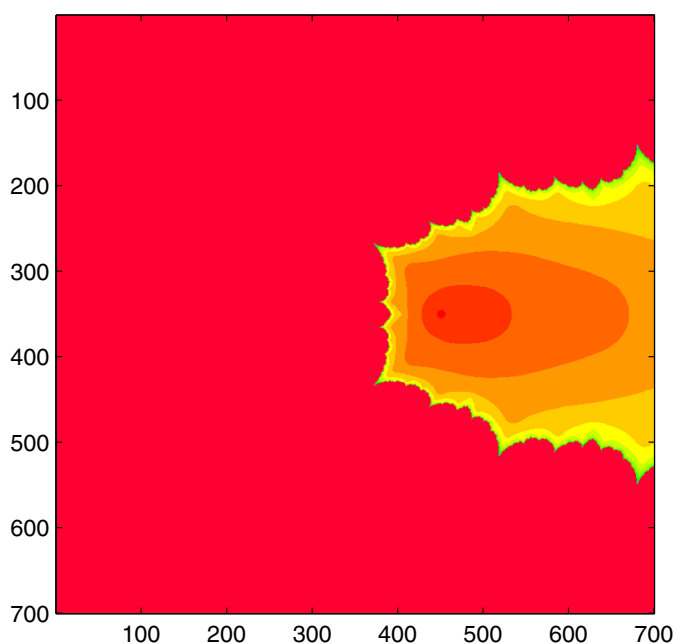

(b) Proposed method (20) at $\lambda=1 / 2$

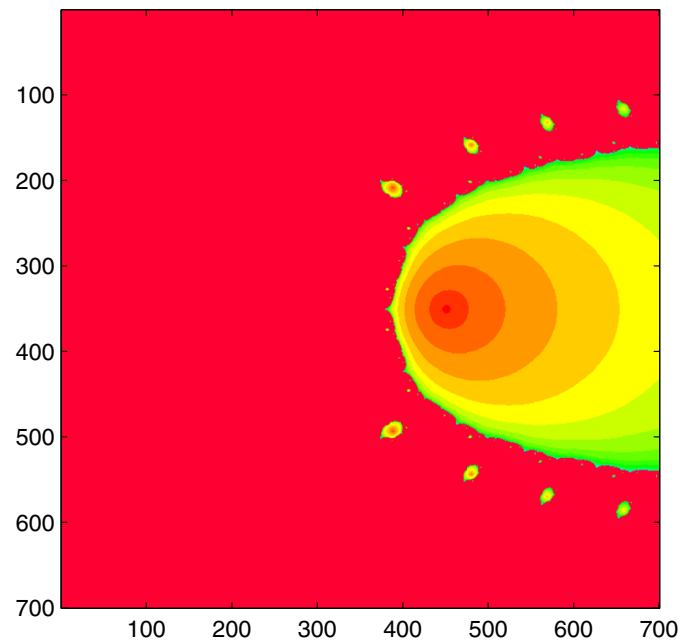

(c) Method by Wu et al. [23]

Fig. 1 Basin of attraction for $F_{1}=\log (z)$ by different methods

which iteration process will flow to every root. In particular, trajectories corresponding to close starting points on the boundary will have divergent orbits. Hence the boundary points of the Julia set describe the instability for any given approximation.

The study of dynamics of the function with the help of an iterative method allows us to study the important information about convergence, divergence, and stability of the method. The basic definitions and dynamical concepts of a rational function can be found in [1]. We apply numerical iterative methods in a square $R \times R=$ $[-3.5,3.5] \times[-3.5,3.5]$ of $700 \times 700$ points with a tolerance- $F\left(z_{n}\right)<5 \times 10^{-2}$ and a maximum of 30 iterations. Under the above conditions starting with every point, $z_{0}$ in the square if the sequence generated by the iterative methods converge to a zero $z^{*}$ of the function, then $z_{0}$ will be in the basin of attraction of this zero and we mark this point $z_{0}$ with a fixed color. Now we describe the basins of attraction for finding complex zeros of our functions $F_{1}=\log (z), F_{2}=\arctan (z)$ and $F_{3}=z+1-\exp (\sin (z))$, by Newton's method (2), proposed derivative-free method (20) and method by Wu et al. [23]. 


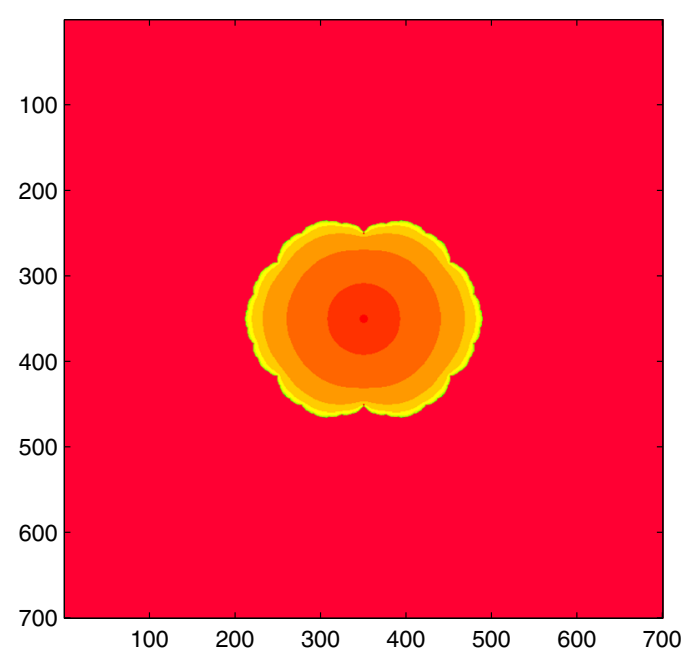

(a) Newton's method (2)

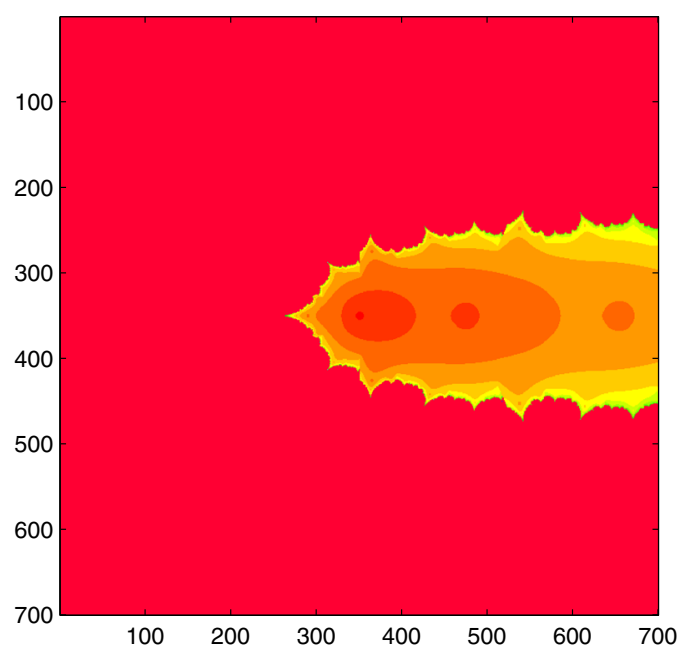

(b) Proposed method (20) at $\lambda=3 / 4$

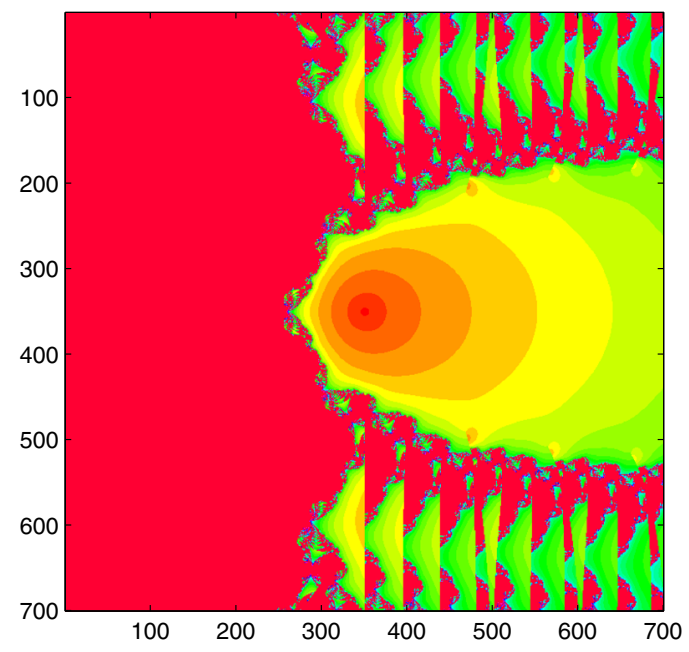

(c) Method by Wu et al. [23]

Fig. 2 Basin of attraction for $F_{2}=\arctan (z)$ by different methods

Note that for the first example $F_{1}=\log (z)$, the basin of the attraction of root is almost the same for Newton's method (2), the proposed method (20), and method by Wu et al. [23]. Numerical results, shown in Table 1 are supported by fractal patterns shown in Fig. 1, clearly basins of attraction by the Newton method (Fig. 1a) end around the point $z_{0}=5$, which confirms that Newton's method diverges at this point. Basins of attraction for the proposed method is nearly rectangular with bigger orbit and dark color showing faster convergence while the basin given by the method of Wu et al. is circular with chaotic behavior and fractal boundaries.

In the second example, $F_{2}=\arctan (z)$, the basin of attraction of each root is larger for the method of Wu et al. [23] and proposed method (20) than Newton's method (2) (Fig. 2), whereas the divergence of Newton method starting with the point $z_{0}=5$ shown earlier (Table 2) is proved in fractal patterns Fig. 2a. Darker color and a bigger rectangle of basins of attraction for the proposed method shows the faster convergence of the method. Again the method of Wu et al. shows some chaotic behavior and fractal boundaries with green color.

For third example $F_{3}=z+1-\exp (\sin (z))$, we have taken a square $R \times R=[-5.5,5.5] \times[-5.5,5.5]$ of $700 \times 700$ points for better results. The basin of attraction of each root in the mentioned region for Newton's 


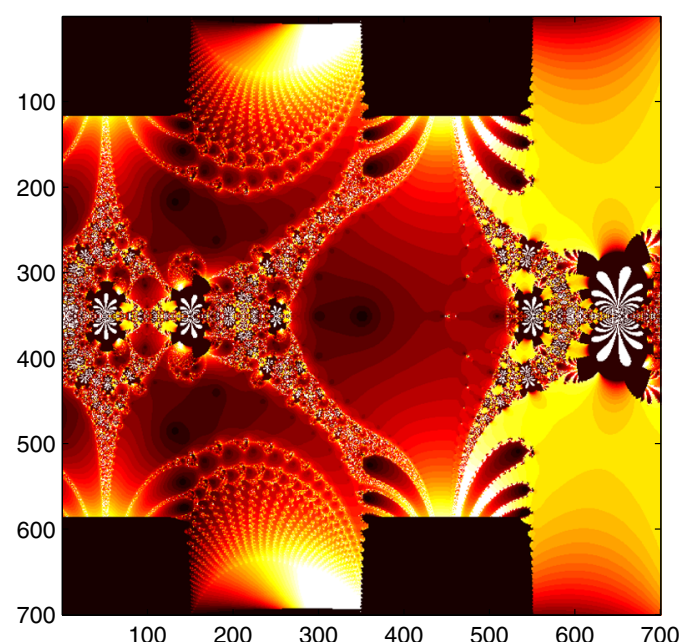

(a) Newton's method (2)

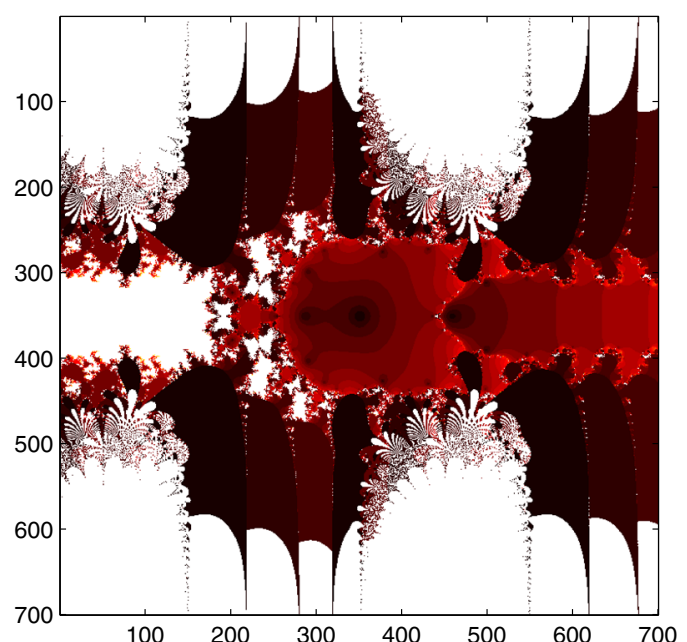

(b) Proposed method (20) at $\lambda=3 / 4$

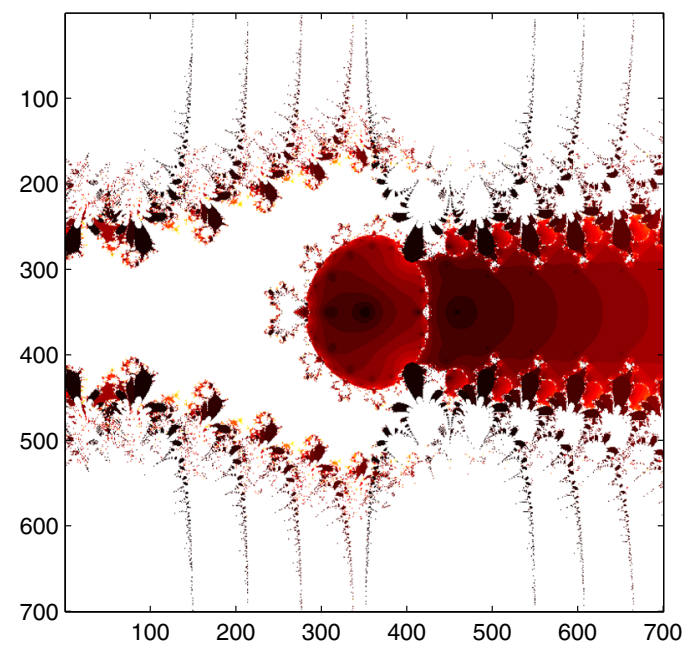

(c) Method by Wu et al. [23]

Fig. 3 Basin of attraction for $F_{3}=z+1-\exp (\sin (z))$ by different methods

method (2) and proposed method (20) is almost the same and larger than the method by Wu et al. [23]. Divergence of Newton's method starting with the point $z_{0}=4$ can be seen in Fig. 3a which is supporting the result in Table 3. All the methods show chaotic behavior with fractal Julia set.

\section{Conclusion}

We discussed a new modified Newton-like method and its different deformations using the drivative and nondrivative terms. Applying the bisection method, we obtained a globally convergent modified Newton's like method which has nice asymptotic quadratic convergence of both the iterative sequence $\left(z_{n}\right)$ and the sequence of the diameters $\left(b_{n}-a_{n}\right)$. Finally, the numerical resuts are supported by the basins of the proposed modified Newton-like method, Newton's method and method by Wu et al. for some selected examples. It looks like that the basins of the method given by Wu et al. have more fractal and chaotic boundaries than those of Newton's method and proposed method. Basins of attraction and fractal boundaries of different methods show how the methods depend on the starting point in a very complicated manner. 
Acknowledgements Authors are thankful to reviewers for their valuable suggestions to improve the manuscript.

Open Access This article is licensed under a Creative Commons Attribution 4.0 International License, which permits use, sharing, adaptation, distribution and reproduction in any medium or format, as long as you give appropriate credit to the original author(s) and the source, provide a link to the Creative Commons licence, and indicate if changes were made. The images or other third party material in this article are included in the article's Creative Commons licence, unless indicated otherwise in a credit line to the material. If material is not included in the article's Creative Commons licence and your intended use is not permitted by statutory regulation or exceeds the permitted use, you will need to obtain permission directly from the copyright holder. To view a copy of this licence, visit http://creativecommons.org/licenses/by/4.0/.

\section{References}

1. Alefeld, G.; Potra, F.A.: Some efficient methods for enclosing zeroes of nonlinear equations. BIT 32, 334-344 (1992)

2. Alefeld, G.; Potra, F.A.; Shi, Y.X.: On enclosing simple roots of nonlinear equations. Math. Comp. 61, 733-744 (1993)

3. Amat, S.; Busquier, S.; Plaza, S.: Review of some iterative root-finding methods from a dynamical point of view. Scientia 10, 3-35 (2004)

4. Benzinger, H.E.: The continuous Newton method. Nucl. Phys. B (Proc. Suppl.) North-Holland, Amsterdam 5A, 327-329 (1988)

5. Ford, W.F.; Pennline, J.A.: Accelerated convergence in Newton's method. SIAM Rev. 38, 658-659 (1996)

6. Gerlach, J.: Accelerated convergence in Newton's method. SIAM Rev. 36, 272-276 (1994)

7. Igarashi, M.: A termination criterion for iterative methods used to find the zeros of polynomials. Math. Comput. 42, 165-171 (1984)

8. Julia, G.: Memoire sure l'iteration des fonction rationelles. J. Math. Pures Appl. 81, 47-235 (1918)

9. Kou, J.: A third-order modification of Newton method for systems of non linear equations. Appl. Math. Comput. 191, $117-121$ (2007)

10. Mandelbrot, B.B.: The fractal geometry of nature. Macmillan. ISBN 978-0-7167-1186-5 (1983)

11. Ortega, J.; Rheinholdt, W.: Iterative Solution of Nonlinear Equations in Several Variables. Academic Press, New York (1970)

12. Ozawa, K.: Some globally convergent iterative method based on the bisection iteration for solving nonlinear. Comput. Math. Appl. 28(6), 83-91 (1994)

13. Ozban, A.Y.: Some new variants of Newton's method. Appl. Math. Lett. 17, 677-682 (2004)

14. Scott, M.; Neta, B.; Chun, C.: Basin attractors for various methods. Appl. Math. Comput. 218(2), 2584-2599 (2011)

15. Singh, M.K.: A Six-order variant of Newton's method for solving non linear equations. Comput. Methods Sci. Technol. 15(2), 185-193 (2009)

16. Singh, M.K.: On an new open type variant of Newton's method. J. Appl. Math. Stat. Inf. 10(2), 21-31 (2014)

17. Steven, M.: Hetzler: a continuous version of Newton's method. Coll. Math. J. 28, 348-351 (1997)

18. Traub, J.F.: Iterative Methods for the Solution of Equations. Prentice Hall, Clifford, NJ (1964)

19. Wang, H.; Liu, H.: Note on a cubically convergent Newton-type method under weak conditions. Acta. Appl. Math. 110, 725-735 (2010)

20. Weerakoon, S.; Fernando, T.G.I.: A variant of Newton's method with accelerated third-order convergence. Appl. Math. Lett. 13(8), 87-93 (2000)

21. Wu, X.Y.: A new continuation Newton-like method and its deformation. Appl. Math. Comput. 112, 75-78 (2000)

22. Wu, X.; Fu, D.: New high-order convergence iteration methods without employing derivatives for solving nonlinear equations. Comput. Math. Appl. 41, 489-495 (2001)

23. Yoneyama, K.: Theory of continuous sets of points. Tohoku Math. 12, 43-158 (1917)

Publisher's Note Springer Nature remains neutral with regard to jurisdictional claims in published maps and institutional affiliations. 